\title{
La performance orthographique à l'articulation école-collège : une approche qualitative des marques de pluriel
}

\author{
Brissaud, Catherine, Cogis, Danièle \& Totereau, Corinne \\ Université Grenoble Alpes \\ Catherine.brissaud@ujf-grenoble.fr \\ Université Paris Ouest Nanterre la Défense \\ dcogis@laposte.net \\ Université Grenoble Alpes \\ Corinne.totereau@ujf-grenoble.fr
}

\begin{abstract}
À l'heure où, en France, le ministère de l'éducation nationale tend à rapprocher la scolarité obligatoire de celle des autres pays de la francophonie du Nord en créant un cycle commun au CM2 (primaire) et à la sixième (secondaire), il semble important d'appréhender les performances des jeunes élèves de ces classes dans un domaine qui se révèle toujours aussi sensible socialement, à savoir l'orthographe grammaticale ${ }^{1}$. Plusieurs recherches ont montré que les performances des élèves, comparées à celles d'autrefois, sont en baisse (MEN-DEP, 1996 ; Manesse, Cogis, 2007 ; Rocher, MEN-DEPP, 2008²). Ces résultats n'ont pas donné lieu, jusqu'à présent, à des recommandations ou des décisions vraiment utiles ${ }^{3}$.
\end{abstract}

Au-delà des polémiques sur un enseignement parfois supposé négligent, on peut penser que la réduction considérable de l'horaire de français a provoqué la diminution du temps consacré à l'orthographe (Gourdet, 2009; Prost, 2012) : au collège, notamment, il est devenu difficile de proposer une durée d'enseignement qui permette encore d'apprendre ou de se perfectionner. Mais on ne s'interroge pas toujours suffisamment sur les effets de la complexité de l'orthographe du français. La question qui se pose alors est de savoir quels facteurs linguistiques font varier la performance orthographique à un moment clé de la scolarité, c'est-à-dire à l'articulation école et collège.

Nous nous focaliserons dans cet article sur les marques de pluriel du nom, de l'adjectif et du verbe au présent de l'indicatif. Ces marques sont au programme de l'école en France dès la classe de cours préparatoire $\left(1^{\mathrm{re}}\right.$ année du primaire, désormais $\left.1 \mathrm{P}\right)$, et de nouveau chaque année jusqu'en classe de sixième ${ }^{4}$.

Pour tenter de dégager ce qu'on peut considérer comme bien établi aujourd'hui et contribuer ainsi aux fondements d'une véritable refondation de l'enseignement de l'orthographe, nous nous proposons de puiser dans les recherches conduites depuis une vingtaine d'années, principalement dans le champ de la linguistique, mais aussi dans celui de la psycholinguistique.

L'enjeu est de comprendre, d'un point de vue linguistique, pourquoi la production des marques de pluriel n'est pas toujours aussi simple qu'on le croit, ou, dit autrement, en quoi les marques de pluriel présentent, en raison de la spécificité de l'orthographe française (Dubois, 1965 ; Catach, 1973a ; 1973b ; 1980), un certain nombre de difficultés que les programmes scolaires méconnaissent et qui ne sont donc sans doute pas travaillées suffisamment à l'école. Notre hypothèse est que ces difficultés linguistiques affectent les performances de tous les élèves, mais qu'elles pèsent plus lourdement sur les élèves de milieu défavorisé.

\section{Enquêtes}

Pourquoi les élèves continuent-ils à faire des erreurs, alors qu'ils ont commencé à apprendre les règles d'accord dès leur première année de scolarité et que ces règles font encore l'objet de leçons au moins jusqu'en sixième année? Nous partirons de deux études récentes : la première a été conduite en 2013 dans trois collèges sociodifférenciés de l'académie de Grenoble (Totereau, Brissaud, Reilhac, Bosse, $2013)^{5}$; la seconde, plus ancienne, date de 2005 et a concerné 25 collèges représentatifs de la France 
métropolitaine (Manesse, Cogis, 2007). Toutes deux confirment l'existence d'une corrélation entre les performances des élèves et le milieu social (Dolla, Establet, 1973 ; Brissaud, 1999) ${ }^{6}$.

Dans ces deux études, une dictée a été utilisée pour évaluer les performances des élèves. Même si on sait depuis longtemps qu'une dictée n'est pas « égale » à une autre (Chartier, 1998), et que, ce qui importe, c'est la capacité à orthographier ses propres textes, cette épreuve permet néanmoins de fournir une photographie de la compétence orthographique à un moment donné. Elle seule assure la présence des éléments qu'on entend étudier. Reproductible, elle présente l'avantage supplémentaire de permettre la comparaison dans le temps et dans l'espace, ce que la production d'un écrit ne permet qu'imparfaitement.

La première dictée («Jo») avait pour objectif de préciser les difficultés que l'accord en nombre représente pour des collégiens scolarisés en réseaux de réussite scolaire ${ }^{7}$. Elle a été proposée à 341 élèves de sixième dans trois collèges sociodifférenciés : le collège A de milieu défavorisé, classé en réseau de réussite scolaire (103 élèves: $40 \%$ d'ouvriers et d'employés, $21 \%$ de sans emploi, $14 \%$ de commerçants, artisans et cadres); le collège $\mathrm{B}$, de milieu socioculturel mixte (167 élèves : $40 \%$ d'ouvriers et d'employés, $39 \%$ de commerçants, artisans et cadres, $13 \%$ de sans emploi) ; le collège $\mathrm{C}$, de milieu plutôt favorisé (71 élèves : $45 \%$ de commerçants, artisans et cadres, $27 \%$ d'ouvriers et employés, $11 \%$ de sans emploi).

La seconde dictée («Les arbres ») est un texte de Fénelon qui avait pour objectif de préciser l'évolution des performances orthographiques d'élèves de 11 à 16 ans, d'une part, entre la fin du XIX $X^{\mathrm{e}}$ siècle et la fin du XX $X^{\mathrm{e}}$ siècle (Chervel et Manesse, 1989); d'autre part, entre 1987 et 2005 (Manesse, Cogis, 2007). Le décalage culturel que représente le discours de Fénelon pour des élèves des $\mathrm{XX}^{\mathrm{e}}$ et $\mathrm{XXI}^{\mathrm{e}}$ siècles, et même du XIX ${ }^{\mathrm{e}}$ siècle, n'a pas échappé aux auteurs des enquêtes, mais la possibilité de comparer les performances sur une telle durée et auprès d'un grand nombre d'élèves l'a emporté sur cet inconvénient majeur. En 2005, elle a été proposée à 2767 élèves du CM2 à la troisième, issus de 25 collèges et de 25 classes de CM2 des mêmes secteurs de recrutement, parmi lesquels cinq collèges et cinq classes de CM2 étaient classés en zone d'éducation prioritaire (ZEP). Pour la présente étude, nous avons extrait les résultats des 545 élèves de $\mathrm{CM} 2$ et des 585 élèves de sixième

Dictée « Jo »: Je vais vous raconter l'histoire d'un gentil petit garçon qui s'appelle Jo. Il habite chez son oncle, un vieux monsieur. Ils vivent dans une cité. Cet enfant possède un don extraordinaire. En effet, ses yeux verts voient beaucoup plus loin et plus précisément que ceux de tout le monde ! Dans ses pupilles qui s'agrandissent et qui brillent, on devine des jumelles microscopiques perfectionnées. ${ }^{8}$

Dictée «Les arbres »: Les arbres s'enfoncent dans la terre par leurs racines comme leurs branches s'élèvent vers le ciel. Leurs racines les défendent contre les vents, et vont chercher, comme par de petits tuyaux souterrains, tous les sucs destinés à la nourriture de leur tige. La tige elle-même se revêt d'une dure écorce, qui met le bois tendre à l'abri des injures de l'air. Les branches distribuent en divers canaux la sève que les racines avaient réunie dans le tronc.

Sur le plan méthodologique, même si on peut postuler l'existence d'interactions entre la production d'une forme lexicale et celle d'une marque de pluriel, on ne peut confondre les deux, ne serait-ce que par les activités d'apprentissage que la méconnaissance de l'une ou de l'autre entraine. Par conséquent, nous ne prenons en compte que la capacité à marquer le nombre. Les marques de pluriel comptabilisées sont $s$ (et sa variante $x$ ) et $n t$ pour le verbe (Dubois, $1965: 41-42$ ). Les élèves crédités d'une marque correcte de pluriel n'ont donc pas nécessairement écrit la forme normée attendue, mais ont écrit, par exemple, jumels pour jumelles ou habres pour arbres, ou encore aggrendissent, perfectionées ou soutérains. De même, on a admis le pluriel en $s$ pour canaux et tuyaux: la marque $x$ n'est en effet qu'une variante contextuelle due à la présence du graphème $a u$ en contexte gauche, $s$ restant la marque prototypique du pluriel dans le système nominal. Comme les élèves ont produit de nombreuses formes telles que canots, tuillos, canaus, tuyaus, qui manifestent clairement la présence du pluriel, la non-prise en compte de ces formes aurait faussé les résultats ${ }^{9}$. Quant au singulier, on sait que, à l'oral comme à l'écrit, il se caractérise par une absence de marque (Dubois, 1965 : 17). C'est cette absence de marque que nous comptabilisons pour montrer, par contraste, les difficultés que posent aux élèves les marques du pluriel. 


\section{Production des marques de pluriel}

\subsection{Le pluriel du nom}

La dictée « Jo » comporte trois noms au pluriel (jumelles, pupilles, yeux). Les résultats révèlent un impact important du milieu social sur la production des marques de pluriel : en effet, si les collèges $\mathrm{B}$ et $\mathrm{C}$ (mixte et favorisé) obtiennent un taux de réussite de $75 \%$ environ $^{10}$, le collège A (défavorisé), avec ses $57 \%$ de marques correctes, accuse un écart de 16 points avec le collège $\mathrm{B}$ et de près de 20 points avec le collège $\mathrm{C}^{11}$.

On ne retrouve pas ce décalage avec les sept noms au singulier de la dictée (cité, don, enfant, garçon, histoire, monsieur, oncle). Les performances sont en effet très élevées dans les trois classes de sixième, puisque le collège qui réussit le moins bien (collège A) obtient un taux de réussite de $95 \%$.

Ces résultats débouchent sur deux constats : d'une part, les marques de pluriel attendues sont moins présentes en milieu défavorisé qu'en milieu mixte ou favorisé ; d'autre part, quel que soit le collège, la différence de performance entre le singulier et le pluriel est importante.

Cependant, si on examine les taux de réussite de chacun des noms au pluriel, une autre différence apparait. Elle oppose yeux à jumelles et pupilles :

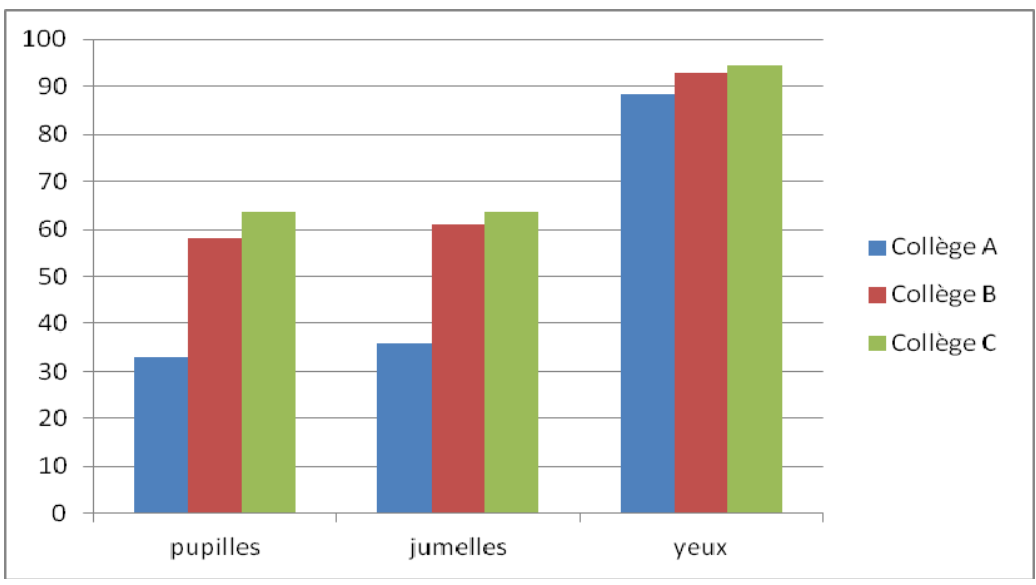

Graphique 1 : Taux de réussite des noms au pluriel en fonction du milieu social (items rangés selon la réussite du collège de milieu défavorisé)

Comme on peut le voir, yeux, non seulement obtient un taux de réussite très élevé, à peu de chose près celui des noms au singulier, mais ce taux est aussi très proche dans les trois collèges. En revanche, jumelles et pupilles sont loin derrière, puisque le taux de marquage le plus élevé n'atteint pas $70 \%$. Ce sont bien ces deux noms qui creusent l'écart entre collèges de milieux différents (30 points séparent le collège $\mathrm{A}$ et $\mathrm{B}$ pour jumelles). Ces résultats débouchent sur un troisième constat : les noms ne sont pas égaux devant le pluriel. La question est alors de savoir si on retrouve de manière systématique ce type de contrastes dans d'autres recherches.

La dictée «Les arbres » comporte onze noms au pluriel (arbres, racines, branches, racines, vents, tuyaux, sucs, injures, branches, canaux, racines) : en classe de sixième, le taux moyen de réussite du marquage du pluriel est de $82 \%$ en Zep et de $86 \%$ hors Zep. Cependant, la différence n'est pas significative ${ }^{12}$. Il faut se tourner vers les performances des élèves de CM2 dans cette même enquête pour trouver et une réussite moyenne plus proche de celle des pluriels de la dictée «Jo » et une différence significative entre milieux sociaux : on relève un écart de 13 points entre les CM2 Zep et non Zep. Ces résultats amènent ainsi à penser que les élèves de Zep ont besoin d'une année supplémentaire pour rattraper en grande partie ceux qui font leur scolarité hors Zep. Pour ce qui est des noms au singulier (terre, ciel, nourriture, tige, écorce, abri, air, sève, tronc), les taux de réussite sont très élevés (94\%), proches de ceux de la dictée « Jo », et ne 
présentent pas de différence significative entre le CM2 et la classe de sixième, quel que soit le milieu social.

Cependant, comme pour la dictée «Jo ", c'est l'examen des taux de réussite de chacun des noms au pluriel qui révèle des différences dans le marquage morphologique. En sixième, le taux moyen de réussite de $84 \%$ masque des taux de réussite contrastés : dans les collèges non Zep, un écart de 26 points sépare l'item le moins bien réussi (canaux) de l'item le mieux réussi (arbres); dans les collèges Zep, cet écart concerne les mêmes noms, mais monte jusqu'à 35 points. Deux éléments apparaissent encore plus nettement ici : d'une part, pour un nom donné, l'écart entre Zep et non Zep est faible, mis à part canaux et 4-racines ${ }^{13}$; d'autre part, quel que soit le milieu social, si on range les différents noms selon leur score, du plus faible au plus élevé, l'ordre des noms reste globalement le même.

Pour aller plus loin, il est intéressant d'examiner les performances en CM2. À première vue, l'ordre de réussite n'y est pas tout à fait le même en Zep et non Zep. En réalité, l'évolution du CM2 à la sixième est marquée par un double mouvement : on observe une augmentation des taux de réussite de tous les items et un resserrement entre les résultats en Zep et non Zep, qui s'accompagnent de quelques permutations locales de rang. Ce qui est en effet tout à fait remarquable, c'est que les différents items se situent dans les mêmes « régions » de l'échelle des taux de réussite et qu'ils évoluent pour l'essentiel à l'intérieur de ces régions, en CM2 comme en sixième, en Zep comme en non Zep :

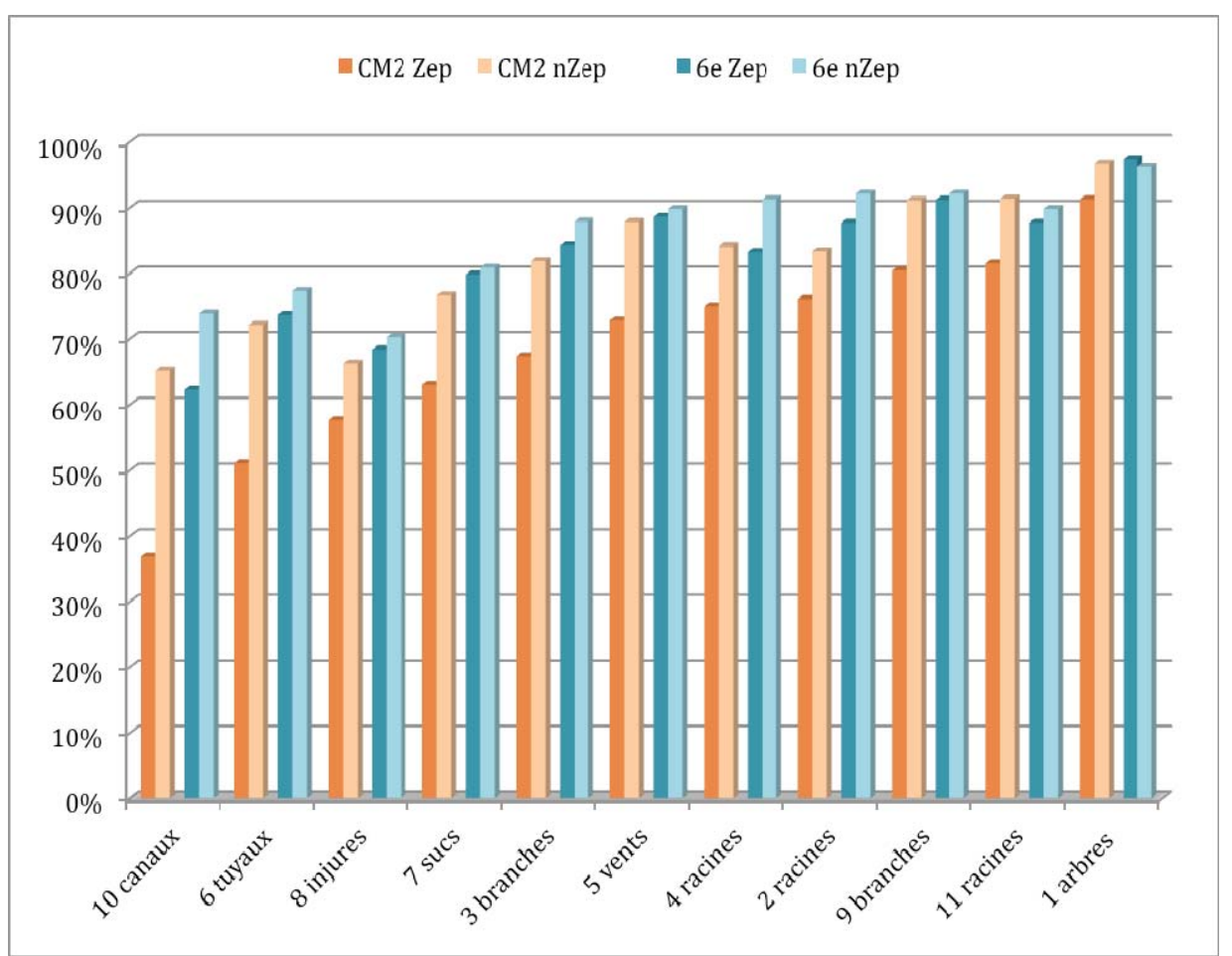

Graphique 2: Taux de réussite des noms au pluriel en fonction de la classe et du milieu social (items rangés selon la réussite des classes de CM2 de milieu défavorisé)

Ainsi, l'examen des taux de marques de pluriel sur les noms dans la dictée «Les arbres » montre que, comme dans la dictée «Jo », ce marquage s'effectue de façon différenciée. Avant d'analyser ce qui peut expliquer ces différences, notamment pour des noms identiques qui n'obtiennent pas le même score, à savoir racines et branches, nous devons aller voir si la situation est aussi contrastée pour les verbes et les adjectifs. 


\subsection{Le pluriel du verbe}

Comme pour le nom, on observe un lien entre milieu social et production du pluriel pour les verbes de la dictée « Jo » : le taux de réussite pour les quatre formes (vivent, voient, s'agrandissent, brillent) varie de $36 \%$ à $72 \%$. Comme pour le nom, on ne retrouve pas un tel décalage avec les verbes au singulier. Pour trois des quatre verbes au singulier (s'appelle, habite, devine), on observe au maximum six points de différence entre le collège qui réussit le mieux (le plus souvent le collège $\mathrm{B}$ ) et le collège qui réussit le moins bien (le collège A). La forme possède, quant à elle, très bien réussie par les élèves de milieu social favorisé, génère néanmoins 18 points d'écart avec ceux du collège défavorisé. Tout se passe comme si cette forme possède se rapprochait des verbes au pluriel en ce qui concerne la difficulté orthographique.

Cependant, comme pour le nom, les variations sont grandes d'un verbe au pluriel à l'autre : un écart de 15 points (pour vivent) à 27 points (pour voient) sépare le collège $\mathrm{A}$ du collège $\mathrm{C} ; 24$ points (pour brillent) à 31 points (pour vivent) éloignent les collèges A et B :

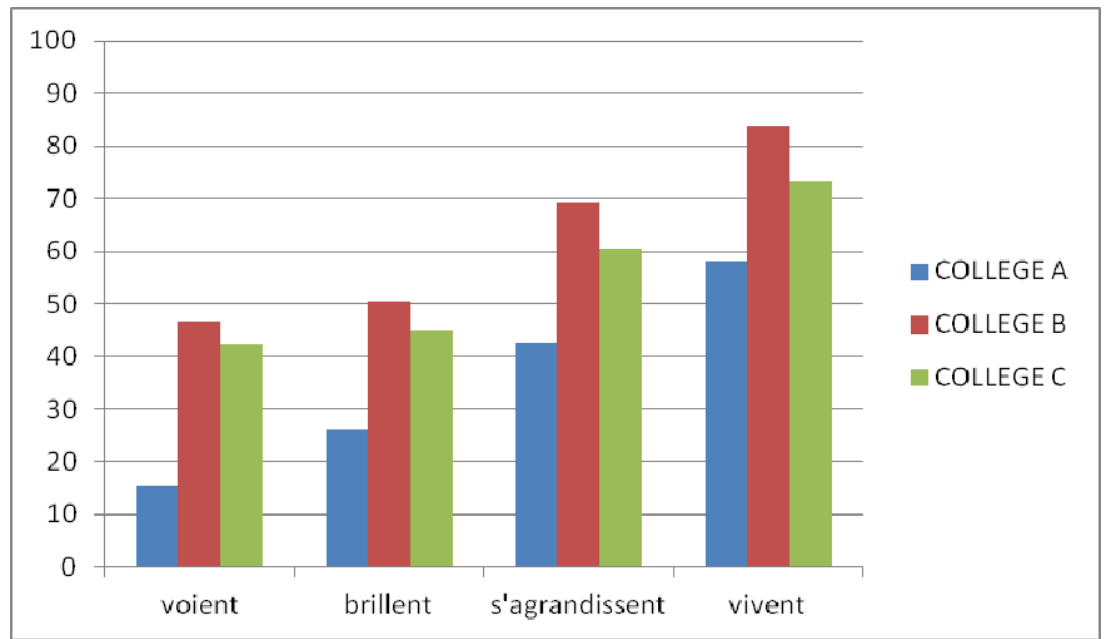

Graphique 3 : Taux de réussite des verbes au pluriel en fonction du milieu social (items rangés selon la réussite du collège de milieu défavorisé)

Mais, ce qui est remarquable, c'est que, comme pour les noms, l'ordre de réussite est conservé, quel que soit le collège. Les marques de pluriel elles-mêmes ne sont pas traitées uniformément : celles de voient et de brillent sont systématiquement moins bien réussies que celles de s'agrandissent et de vivent (près de 43 points de différence entre le score de voient et celui de vivent dans le collège $\mathrm{A}$; 37 dans le collège $\mathrm{B}$ et 31 points dans le collège $\mathrm{C}$ ).

La dictée «Les arbres» nous permet-elle de préciser encore les choses ? Le graphique 4 donne les performances des élèves de CM2 et de sixième en fonction du type d'établissement (Zep/non Zep) pour les cinq verbes de la dictée (distribuent, élèvent, défendent, enfoncent, vont). Le taux de réussite moyen varie de $49 \%$ en Zep à $64 \%$ hors Zep en CM2 et de $69 \%$ en Zep à $72 \%$ hors Zep en classe de sixième, à l'exception notable de vont qui dépasse les $85 \%$ de marques correctes en CM2 Zep et $95 \%$ en sixième Zep. Pour ces verbes au pluriel, on observe un effet du milieu social sur la production du pluriel : les différences sont significatives en CM2, alors qu'elles ne le sont pas en sixième. Comme pour le nom, on ne retrouve pas un tel décalage avec les deux verbes au singulier (met, revêt).

Comme pour le nom, cependant, on relève des différences selon les verbes. En classe de sixième, le décalage entre le verbe le mieux réussi (vont) et le verbe le moins bien réussi (distribuent) est de 53 points en ZEP et de 43 points pour les collèges hors ZEP. Mais la différence entre ZEP et non ZEP pour un verbe donné est faible. Comme pour la dictée «Jo », l'ordre de réussite des verbes reste inchangé, quel que soit le milieu social. 
En classe de CM2, on enregistre des différences significatives entre milieux sociaux, comme on l'a observé pour les noms. La différence entre le verbe le mieux réussi (vont, le même en Zep et non ZEP, comme en classe de sixième) et le verbe le moins bien réussi (distribuent, le même partout) est de 63 points en ZEP et de 53 points en collèges non ZEP. Pour un verbe donné, la différence entre les deux types de collège varie de 10 points (pour distribuent) à 23 points (pour s'élèvent ou enfoncent).

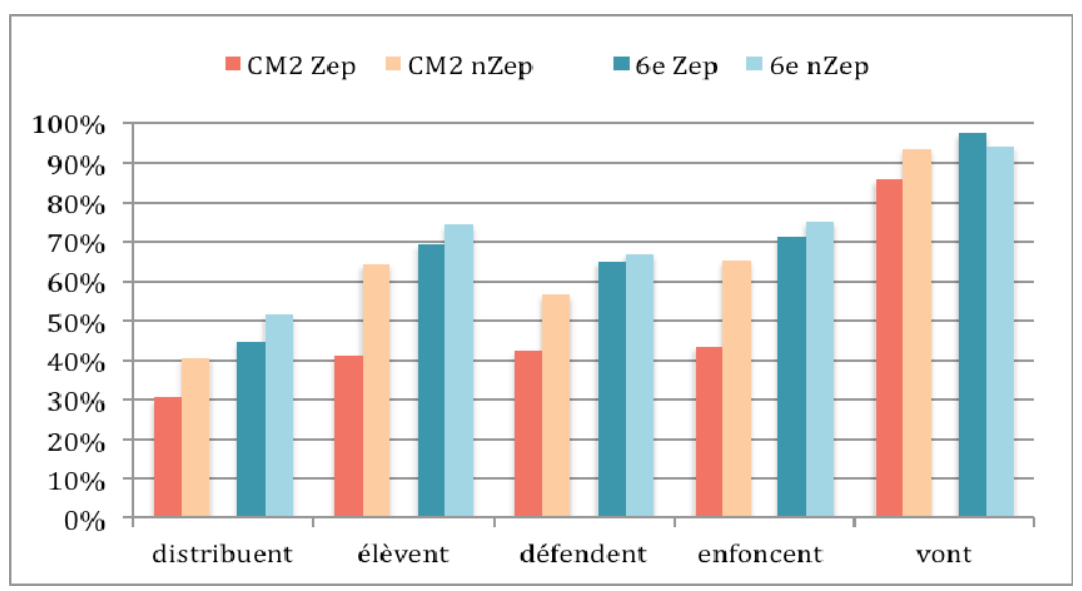

Graphique 4 : Taux de réussite des verbes au pluriel en fonction de la classe et du milieu social (items rangés selon la réussite des classes de CM2 de milieu défavorisé)

L'examen des taux de réussite des verbes au pluriel dans la dictée « Les Arbres » montre que le marquage du pluriel s'effectue de façon différenciée, comme dans la dictée «Jo ». Comme pour le nom, les élèves progressent dans ce marquage d'une classe à l'autre, avec des différences d'un verbe à l'autre.

En fin de compte, si on rassemble les résultats des deux niveaux de classe, Zep et non Zep, on constate que les taux de réussite des écoliers non Zep et des collégiens Zep ou non Zep sont proches, et que l'ordre des réussites pour chaque nom ou chaque verbe obéit généralement à celui de la scolarité (CM2 Zep < $\mathrm{CM} 2$ non Zep $<6^{\mathrm{e}}$ Zep $<6^{\mathrm{e}}$ non Zep).

\subsection{Le pluriel de l'adjectif}

Qu'en est-il du marquage de l'adjectif? La dictée «Jo» comporte trois adjectifs au pluriel (verts, microscopiques, perfectionnées) et quatre adjectifs au singulier (extraordinaire, gentil, petit, vieux ${ }^{14}$ ). Ici encore, c'est bien le marquage du pluriel qui est problématique pour tous les élèves : les taux de réussite, qui vont de $10 \%$ (pour le collège A) à $29 \%$ (pour le collège C) sont particulièrement faibles, bien plus faibles que pour les noms et les verbes. Mais, si la difficulté semble grande dans les trois collèges, elle l'est encore davantage dans le collège A, comme le montre le graphique $5:$ l'impact du milieu social sur la production des marques de pluriel de l'adjectif est sans appel.

On ne retrouve pas une telle difficulté au singulier. Les adjectifs extraordinaire et petit ne sont jamais affectés d'une marque de pluriel; pour gentil, on enregistre seulement deux occurrences en ent et quatre en $s$. Ces résultats rappellent donc ceux des noms et des verbes au singulier. 


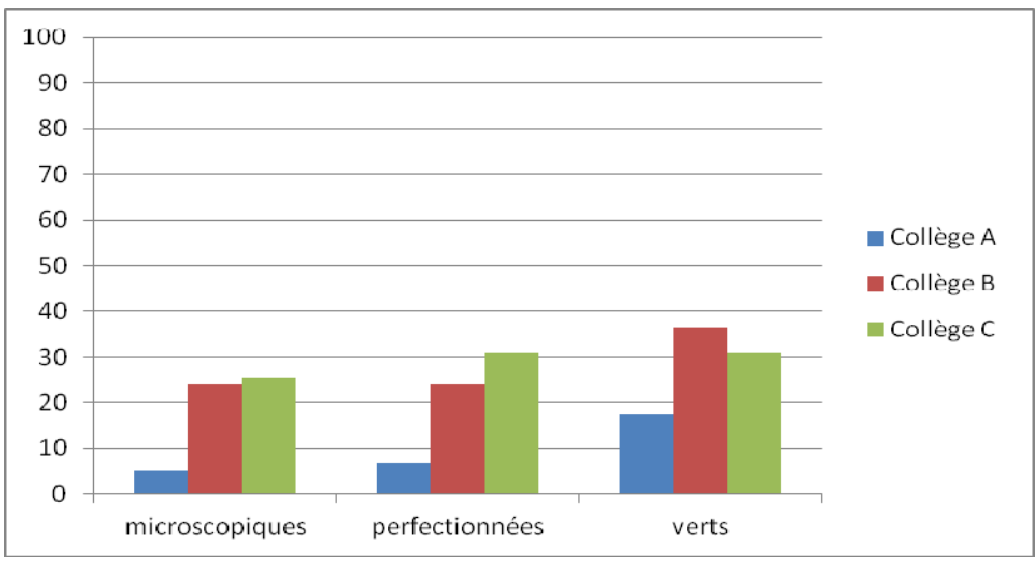

Graphique 5 : Taux de réussite des adjectifs au pluriel en fonction du milieu social (items rangés selon la réussite du collège de milieu défavorisé)

Dans le collège A, l'accord de l'adjectif microscopiques est réalisé dans $5 \%$ des cas, celui de perfectionnées dans $7 \%$ des cas et celui de verts par $18 \%$ des élèves. Comme pour le nom et pour le verbe, les marques de pluriel elles-mêmes ne sont pas traitées uniformément : celles de microscopiques et de perfectionnées sont plus souvent absentes que celle de verts.

La dictée «Les arbres » comporte trois adjectifs au pluriel (souterrains, destinés, petits) et un adjectif au singulier (dure). En classe de sixième, comme en classe de CM2, on observe une différence significative des performances moyennes entre Zep et non Zep. Cet écart se réduit cependant en classe de sixième.

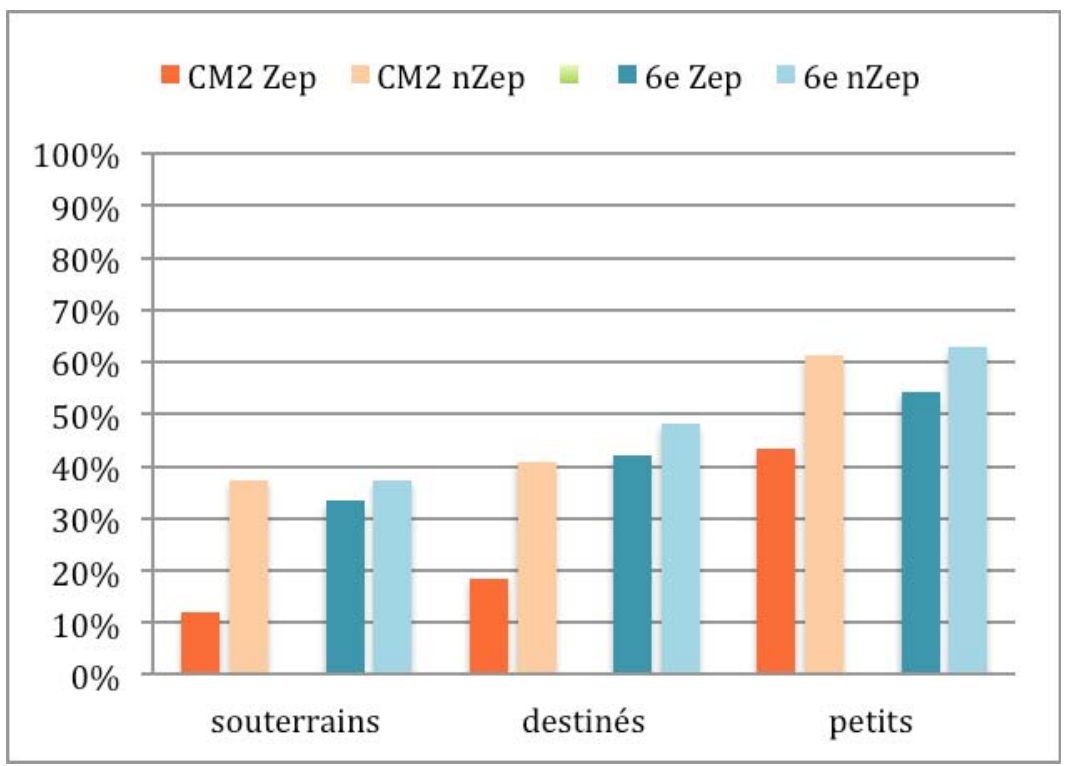

Graphique 6 : Taux de réussite des adjectifs au pluriel en fonction de la classe et du milieu social (items rangés selon la réussite des classes de CM2 de milieu défavorisé)

Si on examine les taux de réussite de chacun des adjectifs au pluriel, il apparait que petits est mieux réussi que souterrains et destinés. Ce décalage est observé, quels que soient le milieu social et le niveau de scolarité.

Ainsi, l'ensemble des données met en évidence une moindre réussite du marquage au pluriel qu'au singulier, sauf cas d'espèces. Mais des différences existent selon les items, ce qui laisse deviner l'existence de facteurs linguistiques à l'œuvre. 


\section{Facteurs de complexité linguistique}

L'enseignement de l'orthographe grammaticale est traditionnellement fondé sur l'apprentissage des règles; corolairement, les fautes sont attribuées au manque de connaissance de ces règles. Mais, si la connaissance ou la méconnaissance des règles était le facteur principal en jeu dans la production des marques de pluriel, alors on ne devrait pas observer des disparités aussi importantes entre items d'une même classe syntaxique, d'une part, ni une telle régularité dans les taux de réussite entre Zep et non Zep, d'autre part. Il ressort en effet de ces enquêtes que ce qui est difficile pour les uns l'est pour les autres et que, quand il n'y a pas de difficulté particulière, les résultats des élèves de milieux défavorisés se rapprochent de ceux des élèves de milieux mixtes ou favorisés. En d'autres termes, il s'agit d'abord de difficultés liées au fonctionnement de l'orthographe française, et, ensuite, on peut le supposer, à une tradition d'enseignement qui, pour l'essentiel, ignore ces difficultés ou ne parvient pas à les traiter de façon efficace.

L'hypothèse qui sera la nôtre dans cette section est qu'un taux de réussite plus faible que les autres dans une catégorie donnée est le signe d'une difficulté orthographique, d'un obstacle linguistique à franchir, et que, inversement, un taux plus élevé que les autres signale un fonctionnement linguistique sans histoire qui ne mérite plus qu'on s'y attarde en classe de CM2 ou de sixième.

\subsection{Contrastes du nombre}

En sixième, voire en CM2 de façon plus nuancée, le singulier ne pose pas de problème; le pluriel en pose, mais de façon différenciée, non seulement selon les classes syntaxiques, mais aussi, de manière généralement méconnue, selon les unités à l'intérieur des classes syntaxiques.

\subsubsection{Le singulier, un nombre chanceux}

Avec le singulier, les élèves courent peu de risque : ils savent s'abstenir de produire une marque de pluriel quand elle n'est pas attendue. Une étude qui mesure les performances d'élèves de CM2 entre 1987 et 2007 , soit à peu près sur la même période que l'enquête «Les arbres ", apporte une confirmation (Rocher, MEN-Depp, 2008) : il n'existe ni baisse des résultats ni obstacle particulier au singulier quand le déterminant est un article défini, par exemple avec le (99\% en 1987/100\% en 2007); soir (99\%/96\%); la (99\%/99\%) maison (98\%/97\%) ; le (99\%/99\%) chien (98\%/98\%). En revanche, quand le déterminant est en concurrence avec un autre déterminant homophone, les taux de réussite sont moins élevés et baissent en 2007, par exemple dans leur (89\%/80\%) chemin (92\%/86\%), comme si la difficulté rencontrée sur le déterminant se répercutait sur le nom.

Un nom, cependant, se remarque dans la dictée «Les arbres », car il déroge au principe d'un singulier sans histoire : $25 \%$ des élèves de CM2 et $28 \%$ de ceux de sixième mettent un $s$ à abri. On peut alors se demander si ce $s$ est une marque de pluriel, ou s'il n'est pas plutôt une lettre d'un autre type, non fonctionnelle comme dans souris, brebis, croquis, débris, ou dérivative comme dans acquis, indécis, soumis, tapis. Cette seconde supposition est corroborée par des commentaires d'élèves en classe ou lors d'entretiens ${ }^{15}$ : ils expliquent souvent qu'ils sont obligés de mettre un $s$ (ou un $t$, un $e$, un $x$ ), parce qu'un mot ne peut pas se terminer par $i$.

La tendance à la réussite au singulier semble être la même pour les verbes. Une recherche menée en 2011 au Québec auprès de 124 élèves de $5^{\mathrm{e}}$ et $6^{\mathrm{e}}$ primaires le confirme : les trois verbes au présent de la dictée «Les ours" (fait, dépasse, manque) sont corrects à $86 \%$ en 5P, et, respectivement à $88 \%, 96 \%, 98 \%$ en 6P (Nadeau, Fisher, Cogis, 2012). Le taux de réussite quelque peu divergent de $60 \%$ environ obtenu par met dans la dictée "Les arbres ", en CM2 comme en sixième, pourrait s'expliquer en partie par la concurrence d'un mot homophone de haute fréquence (mais).

Pour expliquer la réussite des unités au singulier (et parfois au pluriel), par-delà leur appartenance à des classes syntaxiques différentes, il faut rappeler la caractéristique du singulier en français : défini par l'absence de marque de pluriel, il se confond avec la structure phonographique de l'unité lexicale 
(garçon, tige, habite), puisque, contrairement aux autres langues romanes, il n'existe pas de marque en propre du singulier. La seule contrainte réside alors du côté lexical, c'est-à-dire dans la connaissance des phonogrammes qui composent le mot, et, le cas échéant, du morphogramme lexical ou non fonctionnel qui le clôt.

Deux mots au pluriel obtiennent une réussite similaire à celui des mots au singulier, à savoir yeux et vont. La variation singulier/pluriel n'y est sans doute pas appréhendée au niveau de la marque, mais au niveau du mot (œil/yeux; va/vont). Leur taux de réussite les rapproche des mots au singulier : les élèves n'ont pas à analyser la classe syntaxique pour produire la marque pertinente $(x$ ou $n t)$ : la suite de graphèmes fonctionne comme un tout mémorisé, une sorte de forme lexicalisée.

\subsubsection{Le verbe au pluriel, point nodal de la variation morphologique}

La comparaison des taux de réussite du nom et du verbe dans les deux enquêtes met en évidence la difficulté du pluriel verbal. Ces résultats sont conformes à ceux obtenus dans d'autres études, qu'il s'agisse de recherches issues de la psychologie cognitive ou de recherches produites par l'institution ellemême (Totereau, Thévenin, Fayol, 1997 ; MEN-DPD, 2002 ; Rocher, MEN-DEPP, 2008).

Nous nous en tiendrons à quelques rapprochements dans des dictées : 42\% pour brillent dans la dictée « Jo »; 47\% de réussite à l'entrée en sixième pour parfument dans Les roses jaunes parfument le salon (MEN-DPD 2002) ; 41\% de réussite aux évaluations nationales de CM2 en 2011 à l'item qui concernait le marquage en nombre d'une série de verbes (source : MENJVA ${ }^{16}$ ); $49 \%$ de réussite en $5^{\mathrm{e}}$ année et $50 \%$ en $6^{\mathrm{e}}$ année sur invitent dans Mes parents t'invitent dans une recherche suisse (Bétrix-Koehler, 1991). Dans la recherche québécoise précédemment citée, les taux moyens de réussite des verbes au pluriel sont certes plus élevés (58\% en $5 \mathrm{P}$ et $61 \%$ en $6 \mathrm{P}$ en moyenne), mais restent loin du $100 \%$ de réussite qu'exigent les programmes français à l'issue du CE1. Enfin, dans une recherche qui porte sur la production de chaines d'accord au pluriel en $\mathrm{CM} 2$, cette fois non pas en dictée, mais en rédaction, le taux de marquage moyen des verbes au pluriel est de $60 \%$, là où il est de $81 \%$ pour les noms (Cogis, 2013). Le pluriel verbal au présent de l'indicatif constitue bien une zone critique au seuil du collège.

\subsection{Des pluriels plus difficiles que d'autres}

Ce qui frappe dans l'ensemble des résultats disponibles, c'est la constance des résultats quand on examine les unités linguistiques une à une : certains pluriels sont plus difficiles que d'autres, quel que soit le milieu social. Il est alors possible d'en déduire des indices de complexité linguistique, ou de facilitation, qui se répercutent sur la progression des acquisitions.

\subsubsection{Homophonie}

Le premier indice qui permet de présager la réussite relative d'une forme verbale est l'existence de formes phonologiques différenciées aux personnes 3 et 6 (par exemple /vi/ vs /viv/). Dans la dictée « Jo », on observe ainsi une différence importante entre le traitement des verbes voient et brillent, les moins bien réussis dans les trois collèges, et celui de s'agrandissent et vivent, les mieux réussis dans les trois collèges. Seuls ces deux derniers verbes présentent deux formes phonologiques différentes aux personnes 3 et 6 . Cette facilitation pour la production du pluriel verbal liée à l'information morphophonologique a déjà été établie pour l'adulte par Largy et Fayol (2001), qui ont obtenu plus d'erreurs par exemple sur arrivent que sur surgissent. On retrouve de telles différences dans la recherche québécoise citée ci-dessus (Nadeau, Fisher, Cogis, 2012) : peuvent est mieux réussi que mangent et habitent (respectivement $77 \%$ de réussite en $5 \mathrm{P}$ et $82 \%$ en $6 \mathrm{P} ; 66 \%$ de réussite en $5 \mathrm{P}$ et $65 \%$ en $6 \mathrm{P} ; 62 \%$ de réussite en $5 \mathrm{P}$ et $55 \%$ en $6 \mathrm{P}$ ). C'est également le cas dans la recherche également mentionnée, qui porte sur la production de chaines d'accord en rédaction en CM2 (Cogis, 2013) : les «verbes hétérophones » y sont mieux marqués que les « verbes homophones» $(68 \%$ vs $50 \%)$. 
Afin de préciser les effets de l'homophonie, arrêtons-nous sur voient, le verbe de la dictée «Jo » qui entraine le plus d'erreurs dans chacun des trois collèges. La différence pour ce verbe très fréquent est de presque 27 points entre le score du collège $\mathrm{A}$, le plus défavorisé, et celui du collège $\mathrm{B}$, de milieu mixte. Le graphique 7 précise la répartition des réponses des élèves en fonction du collège fréquenté. Les formes alternatives produites par les élèves apportent des informations complémentaires intéressantes. À part la forme voi (produite par 10 élèves sur 341), toutes les formes produites existent à l'écrit. Les élèves du collège A se distinguent par deux types d'erreurs qu'ils commettent deux fois plus que les autres élèves : la production de la forme normée au singulier (voit : $33 \%$ des erreurs) et celle d'une forme en $s$ (vois : $13 \%$ ). Ces élèves seraient donc davantage sensibles à l'éloignement du signal sonore ses yeux, séparé du verbe par l'adjectif, et à l'absence de marquage du nombre sur l'adjectif vert, qui domine, comme on l'a vu plus haut. Cet exemple montre bien les effets de l'homophonie sur la dispersion des réponses.

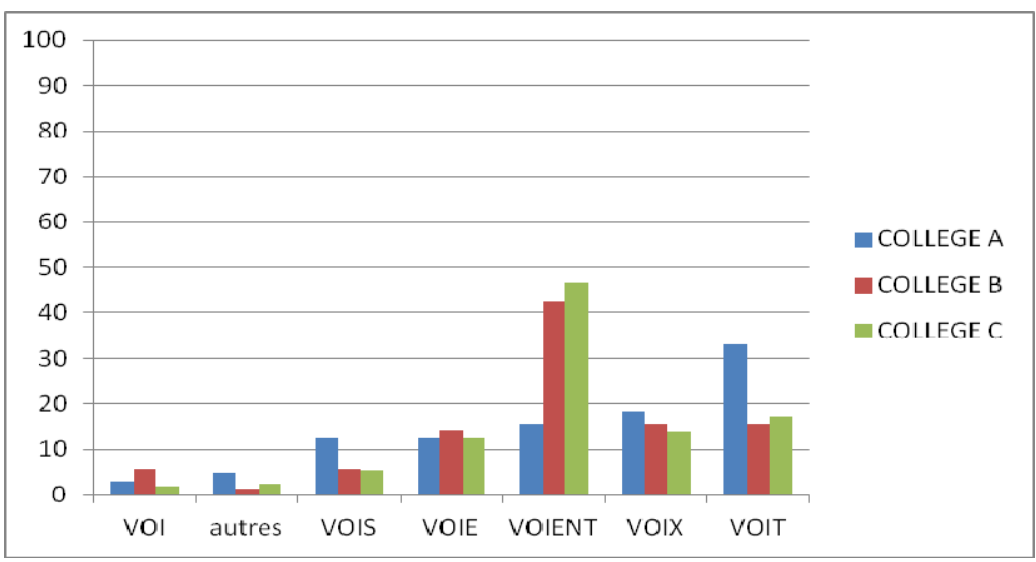

Graphique 7 : Taux de réponse pour chacune des formes produites en fonction du milieu social, rangées selon les réponses du collège de milieu défavorisé

Ainsi, la production d'une forme verbale au pluriel, quand il s'agit d'une forme homophone d'une forme verbale au singulier, ce qui est le cas de tous les verbes en er (hormis aller) et de verbes fréquents comme voir et croire, est problématique aujourd'hui pour un grand nombre d'élèves à l'articulation école-collège.

\subsubsection{Contextes syntaxiques}

Compte tenu de l'avantage que procure l'existence de formes phonologiques différenciées au singulier et au pluriel, on peut s'étonner du relativement faible résultat de défendent dans la dictée «Les arbres » : alors qu'il offre un point d'appui oral, le taux de réussite de la production des marques de pluriel n'atteint pas $70 \%$ en sixième non Zep, à la différence de élèvent et de enfoncent, alors que ces deux formes sont homophones au singulier et au pluriel. Ce résultat inattendu conduit à approfondir l'examen. Effectivement, le pronom les fait écran entre le donneur de marques Leurs racines et défendent. Les élèves semblent traiter la suite pronom + verbe comme une suite déterminant les + nom (les défendes). De nombreuses recherches ont relevé l'impact de la présence de rupteurs (par exemple, Fayol et Largy, 1992 ; Jaffré, Bessonnat, 1993 ; Brissaud, 1998). Dans cette dernière recherche, qui porte sur les formes verbales en /E/, le verbe à l'imparfait espionnait dans la phrase Jacques les espionnait avec des copains est réussi par $48 \%$ des élèves de $\mathrm{CM} 2$ et $56 \%$ des élèves de sixième : les deux erreurs les plus fréquentes sont espionnaient et espionnés, c'est-à-dire des formes qui portent une marque de pluriel correspondant au pluriel du pronom intercalé élevé au statut de donneur de marques.

Ces erreurs sont-elles imputables à des automatismes, à l'instar de celles des « experts » qui peuvent se faire piéger dans des configurations comme Il les timbre (timbres) ou Le chien des voisins arrive (arrivent), ou d'une reconnaissance encore incertaine du sujet, ou encore d'une méconnaissance du pronom objet antéposé, comme il est plus probable, étant donné le niveau de scolarité ? Lors d'entretiens, en effet, des élèves de CM2 n'hésitent pas à pointer un mot précédant le verbe (les ou voisins) pour 
expliquer leur choix du $s$ comme terminaison verbale. On sait que les élèves ont une représentation primitive du sujet comme l'élément qui précède le verbe, ce qui leur suffit dans le cas où il n'existe qu'un seul nom à gauche du verbe, mais provoque des erreurs dans des cas moins favorables (Cogis, Brissaud, 2003).

L'effet du contexte ne concerne pas seulement les verbes: on a vu que le pluriel des noms est généralement réussi, mais il l'est moins quand un déterminant inhabituel vient perturber le jeu. C'est sans doute ainsi qu'on peut expliquer que les taux de réussite varient pour un même nom dans la dictée "Les arbres », notamment en CM2, quand le nom est précédé de les ou de leurs : en fonction du déterminant, branches passe de $67 \%$ de réussite à $80 \%$ en Zep et de 82 à $91 \%$ en non Zep ; quant aux trois occurrences de racines, l'écart entre celles qui sont précédées de leurs ou de les est de l'ordre de 6 à 8 points, sans différence sensible entre Zep et non Zep. On a vu que certains déterminants pouvaient avoir un impact négatif sur des noms au singulier (Rocher, MEN-DEPP, 2008). Les taux de réussite de l'adjectif petits, moins élevés que ce qu'on pouvait attendre, pourraient s'expliquer par une forme de déterminant peu connue des élèves, à savoir de (de petits tuyaux souterrains) et qui n'a donc pas joué le rôle de signal habituel : ces taux plafonnent en effet à $61 \%$ en CM2 et $63 \%$ en sixième non Zep. En Zep, les taux progressent du CM2 à la sixième, mais restent peu élevés (de 43 à 54\%). Le déterminant affecte donc les résultats de tous les élèves, mais de façon plus forte en milieu défavorisé.

On peut encore chercher un effet de contexte quand on examine le type d'erreurs commises dans la dictée « Jo », cette fois sur un verbe au singulier. Les verbes habite et devine ont une forme similaire, puisqu'ils se terminent tous deux par une finale très régulière en français, à savoir consonne $+e$, (histoire, s'appelle, oncle, possède, extraordinaire, monde, terre, nourriture, tige, elle, même, écorce, sève). On observe néanmoins que les deux verbes n'ont pas provoqué les mêmes erreurs : en effet, si le premier n'a entrainé que trois marques de pluriel indues (sur 341), le second en a provoqué huit fois plus (4s et $19 n t$ ). De fait, devine dépend du pronom on, que les élèves traitent régulièrement comme un pluriel, au point d'écrire ont (Brissaud et Cogis, 2011). Certes, il ne s'agit pas d'erreurs massives, mais le contraste doit servir d'alerte pour pousser plus loin l'investigation et le travail en classe.

\section{Conclusion}

L'observation de données issues de différentes recherches permet de conclure qu'il existe indiscutablement des zones de difficulté liées à la production des marques de pluriel en français : si le nom au pluriel est largement réussi, le verbe au pluriel l'est moins, surtout dans le cas où l'on ne peut compter sur une variation phonique brille/brillent vs agrandit/agrandissent) ou dans des contextes syntaxiques non basiques (leurs racines les défendent). Inversement, le singulier ne relève pas de la difficulté orthographique: quand un singulier pose problème, il s'agit le plus souvent d'une forme lexicale particulière ( $a b r i)$ ou d'un environnement spécifique (on devine). Ces difficultés concernent tous les élèves, quel que soit leur milieu social. Elles sont donc bien avant tout d'ordre linguistique. Rappelons que l'orthographe française est considérée comme l'une des plus difficiles au monde (Jaffré et Pellat, 2008).

Une autre conclusion importante se dégage de notre étude. Si la complexité orthographique touche tous les élèves, elle affecte plus fortement les élèves de milieu défavorisé. Sur ce point, les deux enquêtes qui ont servi de point de départ ne concordent pas totalement: la plus récente montre une différence de résultats entre des élèves de sixième scolarisés dans des collèges socialement différenciés, alors que la plus ancienne ne montre cette différence qu'en CM2. Mais ce que cette seconde enquête montre bien, c'est que les élèves de milieu défavorisé rattrapent en grande partie l'écart qui les sépare des autres élèves en classe de sixième. Le décalage selon les milieux n'apparait donc pas rédhibitoire : tous les élèves cheminent et progressent dans le marquage du nombre. Ce constat nous semble devoir être souligné, tant est répandue l'idée que les élèves labellisés «en difficulté » n'apprendraient pas l'orthographe grammaticale (l'orthographe lexicale n'entre pas dans le cadre de cette étude).

Il y a ainsi un véritable enjeu à continuer à travailler l'orthographe non seulement dans le futur cycle des approfondissements (CM1-CM2-6e), mais aussi jusqu'à la fin du collège, en particulier dans les milieux 
les moins favorisés. C'est ce qui semble ressortir du fait que les élèves de Zep n'arrivent au niveau des autres qu'en sixième, du moins dans l'enquête de 2005 qui permet de mesurer la progression d'une année à l'autre. Ces élèves ont assurément besoin de plus de temps que les autres, puisqu'ils sont, plus que les autres, dépendants de l'école pour apprendre. Or l'apprentissage de l'orthographe, en raison de sa complexité, demande beaucoup de temps. La question de la durée impartie à l'enseignement de l'orthographe est donc cruciale. Si cette durée ne cesse de diminuer ${ }^{17}$, la complexité, elle, reste la même.

Toutefois, les difficultés de l'orthographe grammaticale ne peuvent être envisagées indépendamment de la façon dont elles sont traitées en classe. Les méthodes d'enseignement sont effectivement aussi à interroger. On connait la pesante tradition qui emprisonne l'orthographe dans la dictée et ses corrections, sans parvenir à modifier chez de nombreux élèves leurs représentations orthographiques erronées. Aujourd'hui, d'autres dispositifs y parviennent, au moins en partie (Cogis, 2008 ; Geoffre et Brissaud, 2012 ; Brissaud, Cogis, Péret, 2013). Une recherche québécoise a montré tout récemment l'impact positif de deux dispositifs innovants sur les résultats des élèves, et en particulier sur ceux des élèves de milieux défavorisés (Fisher, Nadeau, 2012).

Du côté des programmes relatifs aux accords, il résulte de l'ensemble des recherches que les prescriptions institutionnelles sont problématiques. On pense aux objectifs insoutenables assignés au CE1 des actuels programmes (ou de ceux qui les ont précédés) ${ }^{18}$, mais pas seulement. En effet, concernant l'accord du verbe, ils n'attirent l'attention des enseignants que sur l'inversion du sujet (en CM1) ou sur le sujet qui (en CM2 et à nouveau en sixième), ce qui n'est que la partie visible de l'iceberg! Quid du pronom ils, reprise d'un segment nominal au pluriel, et courant parfois sur plusieurs phrases dans la plupart des textes d'élèves, ou de certains compléments intercalés qui peuvent faire obstacle à l'accord ? Pourquoi continuer à négliger l'homophonie verbale des verbes du $1^{\text {er }}$ groupe, présentés comme "simples», parce que " réguliers ", et donc supposés faciles, alors que certains verbes comme aller, faire, prendre, vivre, grandir, pouvoir, sont plus accessibles que d'autres et peuvent servir de point d'appui aux apprentissages du nombre verbal?

Nous éprouvons aussi, à ce stade de notre réflexion, le besoin de revenir sur le choix des items utilisés pour évaluer l'orthographe grammaticale à l'articulation du primaire et du secondaire. Les noms et les verbes au singulier n'apportent que peu d'informations sur le niveau des élèves : il est plus efficace de s'en tenir au pluriel, mais de sélectionner soigneusement le niveau de complexité des items proposés en fonction des programmes et des capacités cognitives des élèves. Examinons, par exemple, les accords du verbe proposés aux élèves de CM2, en janvier, dans les évaluations nationales de 2011 (source: MENJVA) : la foule se rassemble - les motards de la police passent - ils empêchent - les voitures publicitaires arrivent - les organisateurs de la course annoncent - les coureurs surgissent. On observe un nom collectif évoquant la pluralité (la foule), un verbe dépendant d'une reprise pronominale (ils), deux groupes nominaux composés avec rupteur au singulier (les motards de la police et les organisateurs de la course), et, finalement, un seul verbe avec une opposition marquée entre le singulier et le pluriel et se situant à la fin de la dictée (surgit/surgissent). Comment, dans ces conditions, s'étonner que seulement $41 \%$ des élèves aient réussi à orthographier cinq verbes sur six ? N'était-ce pas prévisible, compte tenu des résultats de la recherche ? On imagine le décalage dans les résultats lié au milieu social des élèves ...

Recherche après recherche, la confrontation des résultats montre bien l'intérêt de se pencher sur des éléments qui se détachent du lot commun dans une production orthographique d'élève, en l'occurrence pour les marques de pluriel, un nom, un adjectif, un verbe, moins bien marqués que ce qui est attendu, parfois mieux. Prendre ces éléments comme des signaux d'alerte permet la plupart du temps de préciser quels sont les obstacles linguistiques sous-jacents. Il est alors possible de tenter de dénouer ces nœuds de difficultés en transformant ces obstacles en espaces spécifiques du travail orthographique. Il y a là une hypothèse didactique forte, nous semble-t-il. Si on travaille de façon plus explicite, plus systématique, plus graduelle, mieux ciblée sur les zones de difficultés identifiées, les progrès peuvent s'accélérer chez tous les élèves, et en particulier chez les élèves trop souvent laissés pour compte en orthographe. 


\section{Références bibliographiques}

Betrix Koehler, D. (1991). Dis-moi comment tu orthographies, je te dirai qui tu es; analyse des performances orthographiques des élèves de $5^{e}$ et de $6^{e}$, Centre vaudois de recherches pédagogiques.

Brissaud, C. (1998). L'imparfait et ses concurrents du CM2 à la troisième, Le français aujourd'hui, 122, 62-70.

Brissaud, C. (1999). La réalisation de l'accord du participe passé employé avec avoir. De l'influence de quelques variables linguistiques et sociales, Langage et société, 88, 5-24.

Brissaud, C., Cogis, D. (2011). Comment enseigner l'orthographe aujourd'hui ? Paris: Hatier.

Brissaud, C., Cogis, D. et Péret, C. (2013). L'enseignement de l'orthographe : une mission encore possible ?, in Baddeley, S., Jejcic, F., Martinez, C., L'orthographe en quatre temps. Paris, Champion, 161-202.

Catach, N. (1973a). «La structure de l'orthographe française », La Recherche, n 39, vol. 4, p. 949-956.

Catach, N. (1973b). «Que faut-il entendre par système graphique du français ? », Langue Française, n 20, p. 30-44.

Catach, N. (1980). L'Orthographe française. Traité théorique et pratique avec des travaux d'application et leurs corrigés, Paris, Nathan, 336 p.

Chartier A.-M. (1998). Épreuves du certificat d'études primaires en 1995. Études de quelques facteurs ayant pu agir sur les résultats des élèves, Éducation et formations, 53, 19-34.

Chervel, A. et Manesse, D. (1989). La dictée. Les Français et l'orthographe, 1873-1987. Paris : INRP/CalmannLévy.

Cogis, D. (2008). Morphographie et didactique, au carrefour des recherches. In Brissaud, C., Jaffré J.-P., Pellat, J.-C., (dir.), Nouvelles recherches en orthographe, Limoges, Editions Lambert Lucas, 181-201.

Cogis D. (2013). Du prescrit au réel en CM2 : l'accord sujet-verbe dans le corpus Grenouille. In Gunnarsson, C. \& Auriac-Slusarczyk, E. (dir.). Écriture et réécriture chez les élèves. Un corpus à la croisée de genres discursifs et des méthodologies d'analyse. Paris : Éd. Academia-Bruylant, Coll. «Sciences du langage. Carrefour et points de vue », 61-84.

Cogis D. et Brissaud C. (2003). L'orthographe : une clé pour l'observation réfléchie de la langue ?, Repères, 28, 47-70.

Dolla A. \& Establet R. (1973) : Un point chaud du système orthographique : les formes en /E/, Bref, n 9, pp. 36-49.

Dubois, J. (1965). Grammaire structurale du français : nom et pronom. Paris, Larousse.

Fayol M. et Largy P. (1992). Une approche expérimentale de l'accord sujet-verbe. Langue française, 95, 80-98.

Fisher, C. et Nadeau, M. (2012). La phrase dictée du jour et la "grammaire nouvelle » : pour des progrès en orthographe grammaticale. La Lettre de l'AIRDF, 52, 31-35.

Fisher, C. et Nadeau, M. (2014). Pratiques innovantes et développement de compétences en orthographe grammaticale. Communication au Symposium organisé par Chantal Ouellet, Congrès WRAB, Paris-Nanterre, 1922 février 2014.

Geoffre, T., Brissaud, C. (2012). L'accord sujet-verbe : acquis en fin d'école primaire, vraiment ?. In éd. Institut de linguistique française, 3e congrès mondial de linguistique française (pp. 287-306). http://dx.doi.org/10.1051/shsconf/20120100196

Gourdet, P. (2009). L'enseignement de la grammaire à l'école élémentaire: le cas du verbe en CE2, Thèse de doctorat, Université Paris Ouest - Nanterre - La Défense.

Jaffré, J.-P. (2003). Les commentaires métagraphiques ». Faits de langues, 22, 67-76.

Jaffré, J.-P.et Bessonnat, D. (1993). Accord ou pas d'accord. Les chaines morphologiques. Pratiques, 77, 25-42. 
Jaffré, J.-P. et Pellat, J.-C. (2008). Sémiographie et orthographes : le cas du français. In Brissaud, C., Jaffré J.-P., Pellat, J.-C., (dir.), Nouvelles recherches en orthographe, Limoges, Editions Lambert Lucas, 9-30.

Largy, P. et Fayol, M. (2001). Oral cues improve subject-verb agreement in written French. International Journal of Psychology, 36 (2), 121-132.

Manesse, D et Cogis, D. (2007). L'orthographe, à qui la faute? Paris, ESF éditeur.

MEN-DEP (1996). Connaissances en français et en calcul des élèves des années vingt et d'aujourd'hui. Les Dossiers d'Éducation \& Formations, 62.

MEN-DPD (2002). Évaluations CE2-sixième. Repères nationaux septembre 2001. Les dossiers, 128, 210-211.

Nadeau, M., Fisher, C. et Cogis, D. (2012). Progression des savoirs sur le verbe : effets de pratiques innovantes. Colloque international Le complexe du verbe, Lyon 30-31 mai 2012.

Prost, A. (30 mai 2012). La réduction du temps de travail des élèves est un formidable gâchis. Le Monde.

Rocher, T., MEN-DEPP (2008). Lire, écrire, compter : les performances des élèves de CM2 à vingt ans d'intervalle 1987-2007, Direction de l'évaluation, de la prospective et de la performance. Note 08.38.

Totereau, C., Brissaud, C., Reilhac, C., Bosse, M.-L. (2013). L'orthographe grammaticale au collège : une approche sociodifférenciée. ANAE, 123, 164-171.

Totereau C., Thévenin M.-G., \& Fayol M., (1997). Acquisition de la morphologie du nombre à l'écrit en français » In L. Rieben, M. Fayol, \& C. Perfetti (Ed.), Des orthographes et leur acquisition, Lausanne-Paris, Delachaux et Niestlé.

${ }^{1}$ Nous remercions très sincèrement les deux évaluateurs attentifs qui nous ont poussées à clarifier un certain nombre de points.

${ }^{2}$ La direction de l'évaluation, de la prospective et de la performance (DEPP) est un service du ministère de l'éducation nationale et du ministère de l'enseignement supérieur et de la recherche chargé des études statistiques. Sa dénomination varie dans le temps (DEP, direction de l'évaluation et de la prospective; DPD, direction de la programmation et du développement).

${ }^{3}$ La circulaire $n^{\circ} 2012-067$ du 27-4-2012 (Bulletin officiel $n^{\circ} 18,3$ mai 2012) fait la recommandation suivante aux professeurs : «L'orthographe doit faire l'objet d'un enseignement structuré, organisé et progressif qui s'appuie sur des leçons spécifiques et régulières chaque semaine tout au long de la scolarité élémentaire et poursuivies au collège ». On souhaiterait davantage de précision...

${ }^{4}$ Les programmes de 2008, les derniers parus à ce jour, stipulent pour le cours préparatoire : "Commencer à utiliser de manière autonome les marques du genre et du nombre (pluriel du nom, féminin de l'adjectif, terminaison -nt des verbes du 1er groupe) ». Ces accords sont ensuite présentés séparément. L'accord dans le groupe nominal est présent dans les programmes de $2^{\mathrm{e}}$ année («marquer l'accord dans le groupe nominal simple, marquer l'accord de l'adjectif qualificatif avec le nom qu'il qualifie »); il est aussi au programme de la $3^{\mathrm{e}}$ année ( Connaitre les règles de l'accord entre déterminant et nom, nom et adjectif) et de la $4^{\mathrm{e}}$ année ("Connaitre la règle de l'accord de l'adjectif (épithète ou attribut) avec le nom »). Il n'est pas mentionné pour la $5^{\mathrm{e}}$ année, mais ressurgit dans les programmes de sixième («les accords dans le groupe nominal (l'adjectif, le déterminant, le nom)»). L'accord du verbe est présent dans les programmes de la $2^{\mathrm{e}}$ année ( «marquer l'accord entre le sujet et le verbe dans les phrases où l'ordre sujet-verbe est respecté »), de la $3^{\mathrm{e}}$ année ( «Connaitre les règles de l'accord du verbe avec son sujet »), de la $4^{\mathrm{e}}$ année ("Appliquer la règle de l'accord du verbe avec son sujet, y compris pour les verbes à un temps composé, et pour les sujets inversés »), de la $5^{\mathrm{e}}$ année («Appliquer la règle de l'accord du verbe avec son sujet, y compris avec le sujet qui de $3^{\mathrm{e}}$ personne »), et de la sixième (l'accord simple sujet-verbe et sujet-attribut ; Appliquer la règle de l'accord du verbe avec son sujet, y compris avec le sujet qui de $3^{\mathrm{e}}$ personne).

${ }^{5}$ Cette étude a bénéficié d'un soutien financier de l'ANR (projet blanc ORTHOLEARN dirigé par S. Valdois) et du CNRS pour la récolte et l'analyse des données effectuées par C. Reilhac et M-L. Bosse.

${ }^{6}$ Les résultats de la dernière enquête Pisa parus en 2013 montrent, pour la France, une "aggravation des déterminismes sociaux » (http://www.education.gouv.fr/cid75515/communication-en-conseil-des-ministres-les- 
resultats-de-l-enquete-pisa.html); à l'échelle internationale, il a été établi un lien fort entre origine sociale et performances en mathématique, même si ce lien n'est pas systématique (http://www.oecd.org/pisa/pisaproducts/pisainfocus/PISA-in-Focus\%20-n36-(fra)-Final.pdf). Sites consultés le 10 mars 2014.

${ }^{7}$ Il n'est pas toujours possible d'obtenir des renseignements précis d'ordre socioprofessionnel. Nous considèrerons donc comme indicatrices d'un milieu populaire ou défavorisé, avec toutes les limites dues aux imprécisions de ces qualificatifs, les mentions institutionnelles RRS (réseau de réussite scolaire) ou, dans des recherches plus anciennes, celle de ZEP (zone d'éducation prioritaire).

${ }^{8}$ Ce texte de dictée a été élaboré à partir du test Repérage Orthographique Collectif (ROC), établi par Michel Zorman et ses collaborateurs.

${ }^{9}$ Dans une perspective de rationalisation de l'orthographe française, il serait d'ailleurs souhaitable de cesser de perpétuer l'erreur d'interprétation de l'abréviation moyenâgeuse $a x$ et d'unifier les marques de pluriel au profit du seul s (tuiaus dans Le Roman de la rose, XIII siècle, d'après Antidote de Druide).

${ }^{10}$ Pour alléger la lecture, nous arrondissons les pourcentages dans le corps du texte.

${ }^{11}$ Cette différence est significative. Pour les tests statistiques de la dictée « Jo », voir Totereau et al. (2013).

${ }^{12}$ Les données de la dictée «Les arbres » ont été traitées selon la méthode de régression logistique. Merci à Pablo Cogis qui s'est chargé des études statistiques.

${ }^{13}$ Dans la mesure où les noms racines et branches figurent respectivement à trois ou deux reprises dans la dictée, nous indiquons un numéro d'ordre d'apparition dans le texte quand c'est nécessaire.

${ }^{14}$ Vieux sort du cadre de cette étude dans la mesure où il ne présente pas de variation singulier/pluriel.

${ }^{15}$ Pour plus d'information sur la méthodologie des entretiens métagraphiques, voir Jaffré (2003).

${ }^{16}$ MENJVA : ministère ministre de l'Éducation nationale, de la Jeunesse et de la Vie associative.

${ }^{17}$ Voir, par exemple, Claude Lelièvre (2008) : http://blogs.mediapart.fr/blog/claude-lelievre/070608/temps-scolairearretons-la-catastrophe (consulté le 18 mars 2014). On notera que les élèves qui ont participé à l'enquête de 2013 avec la dictée « Jo », dont les résultats ont pu paraitre moins bons que ceux des élèves de 2005 , sont entrés au CP en septembre 2008 au moment de la mise en place de la semaine de 24 heures sur quatre jours. Ils ont donc fait toute leur scolarité avec un horaire moindre que celui qu'ont connu leurs prédécesseurs. Ce qui ne laisse pas d'inquiéter : une enquête récente auprès des professeurs de français montre que l'enseignement de l'orthographe trouve difficilement sa place au collège (d'après une enquête menée par une commission à l'initiative du Bureau des programmes d'enseignement du ministère de l'éducation nationale et avec l'aide de la DEPP : une partie a été publiée dans la Note d'information - $\mathrm{N}^{\circ} 13.35$ - décembre 2013, sous le titre Grammaire, orthographe, lexique : quelles pratiques d'enseignement de la langue au collège et en CM2 ? http://www.education.gouv.fr/cid76058/grammaireorthographe-lexique-quelles-pratiques-d-enseignement-de-la-langue-au-college-et-en-cm2.html).

${ }^{18}$ Voir note 4. 